\title{
ABSOLUTELY CONTINUOUS EMBEDDINGS BETWEEN SPACES OF FUNCTIONS
}

\author{
Pedro Fernández-Martínez, Antonio Manzano
}

\begin{abstract}
Absolute continuity of an embedding between Banach function spaces is an interesting property which is closely related to compactness. In this paper we study absolutely continuous embeddings between arbitrary Banach spaces intermediate with respect to the couple $\left(L_{1}(\Omega), L_{\infty}(\Omega)\right)$. Our results allow to check if an embedding of such spaces is absolutely continuous. Applications related with the degree of proximity between two function spaces are established for the case $\Omega=[0,1]$ and $\Omega=[0, \infty)$.
\end{abstract}

Keywords: absolutely continuous embedding, interpolation, quasiconcave function, Banach lattice, proximity between function spaces.

\section{Introduction}

Separable Banach function spaces enjoy a compactness criterion that characterizes compactness of a set of functions as compactness in measure besides absolute equicontinuity of the norms (see [17], [3]). Taking this into account, when it comes to establish compactness of an embedding $Y \hookrightarrow X$ between function spaces it suffices to prove that the closure in $X$ of $B_{Y}$, the closed unit ball of $Y$, is compact in measure and that the elements of $B_{Y}$ have the so-called Absolute Equicontinuity Norm Property in $X$, that is to say,

$$
\forall \varepsilon>0, \exists \delta>0 \text { such that }\left\|f \chi_{D}\right\|_{X}<\varepsilon, \quad \text { for all } f \in B_{Y},
$$

if the measure of $D$ is less than $\delta$. When condition (1.1) is satisfied we say the embedding $Y \hookrightarrow X$ is absolutely continuous (see Definition 2.1 in Section 2).

The two authors were partially supported by Ministerio de Ciencia e Innovación (MTM201015814). The first author was partially supported by Región de Murcia, Fundación Séneca 08791/PI/08.

2010 Mathematics Subject Classification: primary: 46E30; secondary: 46B70, 46B42 
This relationship between compactness and absolute continuity of an embedding $Y \hookrightarrow X$ led Evgeniy Pustylnik and the present authors to investigate absolutely continuous embeddings between rearrangement invariant spaces in [11]. There we worked on the interval $[0,1]$, with the Lebesgue measure, and established certain properties of absolutely continuous embeddings connected with the fundamental functions of the corresponding spaces.

In the present paper we continue the study of absolutely continuous embeddings between function spaces, but this time considering a more general context. As known, a classical result from interpolation theory says that any rearrangement invariant space is an intermediate space with respect to the couple $\left(L_{1}, L_{\infty}\right)$ (see [12], Theorem II.4.1). Then, a natural question is to investigate absolutely continuous embeddings between more general spaces of functions, such as arbitrary intermediate spaces with respect to $\left(L_{1}, L_{\infty}\right)$ (for instance, Banach lattices which are not necessarily rearrangement invariant spaces). This is the aim of this paper. The results we now present extend those given in [11].

Unlike the possibilities we had in [11], in the general setting of intermediate spaces we cannot make use of the good properties of rearrangement invariant spaces. In particular, such a useful tool as the fundamental function is not available. To overcome this difficulty we use techniques based on interpolation theory. Thus, we work with two functions defined for any intermediate space $A$ with respect to a Banach couple $\left(A_{0}, A_{1}\right), \psi$ and $\rho$, which allow to describe in some sense the "position" of $A$ within the couple $\left(A_{0}, A_{1}\right)$. For the relevant couple $\left(L_{1}, L_{\infty}\right)$ the functions $\psi$ and $\rho$, associated to an intermediate space with respect to this couple, can be explicitly computed by using characteristic functions (see [10], Theorem 3.2, and also [8], Lemmata 2.3 and 2.1).

The paper is organized as follows. We start by recalling some basic notions about function spaces and interpolation theory, as well as the precise definition of the functions $\psi$ and $\rho$, in Section 2. The concept of absolutely continuous embedding is given there too. In sections 3 and 4 we investigate the absolutely continuous embeddings between general intermediate spaces with respect to the couple $\left(L_{1}(\Omega, \mu), L_{\infty}(\Omega, \mu)\right)$, where $(\Omega, \mu)$ is a $\sigma$-finite non-atomic measure space. In particular, we give either necessary or sufficient conditions for the absolute continuity of these embeddings in terms of functions $\psi$ and $\rho$. These conditions, formulated by means of different quotients of the functions $\psi$ and $\rho$, provide simple results that allow to check if an embedding is absolutely continuous easily. We illustrate it with some examples. In Section 5, we give a quantitative estimate of the degree of absolute continuity of an embedding $Y \hookrightarrow X$, when $X$ and $Y$ are Banach lattices intermediate for the couple $\left(L_{1}, L_{\infty}\right)$. Finally, in Section 6 , we use our results about absolutely continuous embeddings to draw some conclusions concerning the degree of proximity between arbitrary intermediate spaces for the couple $\left(L_{1}(\Omega), L_{\infty}(\Omega)\right)$, when $\Omega=[0,1]$ and $\Omega=[0, \infty)$. In both cases, it is shown that if two intermediate spaces, $Y \hookrightarrow X$, are not "very close together" then the embedding is absolutely continuous. On the contrary, if the embedding $Y \hookrightarrow X$ is not absolutely continuous, and the corresponding indices of spaces are equal, then $X$ and $Y$ are "neighboring" spaces. 


\section{Preliminaries}

Let $\left(A_{0}, A_{1}\right)$ be a Banach couple, that is, $A_{0}$ and $A_{1}$ are Banach spaces which are continuously embedded in some Hausdorff topological vector space. For $t>0$, the Peetre $K$-functional is the norm on $A_{0}+A_{1}$ given by

$$
K(t, a)=K\left(t, a ; A_{0}, A_{1}\right)=\inf \left\{\left\|a_{0}\right\|_{A_{0}}+t\left\|a_{1}\right\|_{A_{1}} ; a=a_{0}+a_{1}, a_{i} \in A_{i}\right\}
$$

and the $J$-functional is the norm on $A_{0} \cap A_{1}$ defined by

$$
J(t, a)=J\left(t, a ; A_{0}, A_{1}\right)=\max \left\{\|a\|_{A_{0}}, t\|a\|_{A_{1}}\right\} .
$$

We will denote the usual norms on $A_{0}+A_{1}$ and $A_{0} \cap A_{1}$ as $\|\cdot\|_{A_{0}+A_{1}}$ and $\|\cdot\|_{A_{0} \cap A_{1}}$, instead of $K(1, \cdot)$ and $J(1, \cdot)$, respectively.

We say that a Banach space $A$ is intermediate with respect to the couple $\left(A_{0}, A_{1}\right)$ if $A_{0} \cap A_{1} \hookrightarrow A \hookrightarrow A_{0}+A_{1}$, where $\hookrightarrow$ means continuous inclusion. Given an intermediate space $A$ with respect to a couple $\left(A_{0}, A_{1}\right)$, we have two functions that describe the "position" of $A$ within the couple $\left(A_{0}, A_{1}\right)$ (see [5] for more information):

$$
\psi_{A}\left(t ; A_{0}, A_{1}\right)=\sup \left\{K(t, a) ;\|a\|_{A}=1\right\}
$$

and

$$
\rho_{A}\left(t ; A_{0}, A_{1}\right)=\inf \left\{J(t, a) ; a \in A_{0} \cap A_{1},\|a\|_{A}=1\right\} .
$$

These functions are variants of functions studied by Dmitriev [9], Pustylnik [16] or Maligranda and Mastylo [13], among others. Clearly, the functions $\psi_{A}$ and $\rho_{A}$ are strictly positive and non-decreasing, and the functions $\psi_{A}(t) / t$ and $\rho_{A}(t) / t$ are non-increasing. Hence, if we put $\psi_{A}(0)=\rho_{A}(0)=0$, we obtain two quasiconcave functions. Recall that each quasiconcave function has its least concave majorant (see, for example, [3], Proposition 2.5.10).

Given a $\sigma$-finite non-atomic measure space $(\Omega, \mu)$, we shall consider Banach spaces of measurable functions on $\Omega$ which are intermediate spaces with respect to the couple $\left(L_{1}(\Omega), L_{\infty}(\Omega)\right)$. Note that the $K$ - functional for this particular couple can be easily computed through the following well-known equality (see [4], Theorem 5.2.1):

$$
K\left(t, f, L_{1}(\Omega), L_{\infty}(\Omega)\right)=\int_{0}^{t} f^{*}(s) d s
$$

$t>0$ and $f \in L_{1}(\Omega)+L_{\infty}(\Omega)$. Here $f^{*}$ stands for the non-increasing rearrangement of $f$.

Sometimes we shall ask the intermediate space $A$ to be a lattice, that is, whenever $|g| \leqslant|f| \mu$-a.e. with $f \in A$ and $g$ a measurable function, then $g \in A$ and $\|g\|_{A} \leqslant\|f\|_{A}$.

The associated space of $A$ will be denoted by $A^{\prime}$. We recall that $A^{\prime}$ is formed by all (classes of) measurable functions $g$ on $\Omega$ for which

$$
\|g\|_{A^{\prime}}=\sup \left\{\int_{\Omega}|f g| d \mu ;\|f\|_{A} \leqslant 1\right\}<\infty .
$$


The associated space will be termed a norming subspace of $A^{*}$, the dual space of $A$, whenever

$$
\|f\|_{A}=\sup \left\{\int_{\Omega}|f g| d \mu ; g \in A^{\prime},\|g\|_{A^{\prime}}=1\right\}, \quad \text { for all } f \in A .
$$

If $A^{\prime}=A^{*}$ or $A=A^{\prime \prime}$, then $A^{\prime}$ is a norming subspace of $A^{*}$.

We also recall that a Banach lattice $A$ is called a rearrangement invariant space if when $f \in A$ and $g$ is equimeasurable with $f$, then $g \in A$ and $\|f\|_{A}=\|g\|_{A}$. If $A$ is a rearrangement invariant space, the function

$$
\varphi_{A}(t)=\left\|\chi_{D}\right\|_{A}, \quad \text { where } D \subset \Omega \text { with } \mu(D)=t,
$$

is said to be the fundamental function of $A$. It turns out that

$$
\varphi_{A}(t)=\frac{t}{\psi_{A}\left(t ; L_{1}(\Omega), L_{\infty}(\Omega)\right)}=\frac{t}{\rho_{A}\left(t ; L_{1}(\Omega), L_{\infty}(\Omega)\right)} .
$$

In particular, we will use later that

$$
\psi_{L_{1}(\Omega) \cap L_{\infty}(\Omega)}(t)=\rho_{L_{1}(\Omega) \cap L_{\infty}(\Omega)}(t)=\min \{1, t\}
$$

and

$$
\psi_{L_{1}(\Omega)+L_{\infty}(\Omega)}(t)=\rho_{L_{1}(\Omega)+L_{\infty}(\Omega)}(t)=\max \{1, t\} .
$$

Among all rearrangement invariant spaces, Lorentz and Marcinkiewicz spaces will be specially important for us. Given a quasiconcave function $\varphi$ defined on $[0, \infty)$, the Marcinkiewicz space $M_{\varphi}$ is defined as the collection of all (classes of) $\mu$-measurable functions $f$ on $\Omega$ such that

$$
\|f\|_{M_{\varphi}}=\sup _{0<t<\infty}\left\{\frac{\varphi(t)}{t} \int_{0}^{t} f^{*}(s) d s\right\}<\infty .
$$

Analogously, given a positive concave function $\varphi$ defined on $[0, \infty)$ with $\varphi(0)=0$, the Lorentz space $\Lambda_{\varphi}$ is formed by all (classes of) $\mu$-measurable functions on $\Omega$ which have finite norm

$$
\|f\|_{\Lambda_{\varphi}}=\lim _{t \rightarrow 0} \varphi(t)\|f\|_{L_{\infty}}+\int_{0}^{\infty} f^{*}(s) \varphi^{\prime}(s) d s .
$$

When $\varphi$ is concave, the Lorentz space $\Lambda_{\varphi}$ and the Marcinkiewicz space $M_{\varphi}$ are, respectively, the smallest and the biggest rearrangement invariant spaces with fundamental function $\varphi$. More precisely, for any rearrangement invariant space $A$ with fundamental function $\varphi$, it holds that

$$
\Lambda_{\varphi} \hookrightarrow A \hookrightarrow M_{\varphi}
$$

More details on function spaces can be found in the books [3] and [12].

Next we present two results established by Fernández-Cabrera in [10], Theorem 3.2 (see also [8], Lemmata 2.3 and 2.1), that will be useful in the sequel. 
Namely, given a $\sigma$-finite non atomic measure space $(\Omega, \mu)$ and an intermediate space $A$ with respect to $\left(L_{1}(\Omega), L_{\infty}(\Omega)\right)$, for any $0<t<\mu(\Omega)$, it holds that

$$
\psi_{A}(t)=\psi_{A}\left(t ; L_{1}(\Omega), L_{\infty}(\Omega)\right)=\sup \left\{\left\|\chi_{D}\right\|_{A^{\prime}} ; \mu(D) \leqslant t\right\},
$$

and

$$
\rho_{A}(t)=\rho_{A}\left(t ; L_{1}(\Omega), L_{\infty}(\Omega)\right) \sim \frac{t}{\sup \left\{\left\|\chi_{D}\right\|_{A} ; \mu(D) \leqslant t\right\}} .
$$

If, in addition, $A^{\prime}$ is a norming subspace of $A^{*}$, then (2.5) turns out to be an equality.

We note that the techniques used in [10], Theorem 3.2, also apply for a general intermediate Banach space.

In this paper we are interested in studying absolutely continuous embeddings between intermediate spaces with respect to the couple $\left(L_{1}(\Omega), L_{\infty}(\Omega)\right)$. We shall pay special attention to the cases $\Omega=[0,1]$ and $\Omega=[0, \infty)$, with the usual Lebesgue measure (that will be denoted by mes).

We finish this section stating the definition of absolutely continuous embedding.

Definition 2.1. Given two Banach spaces $X$ and $Y$ intermediate with respect to the couple $\left(L_{1}(\Omega), L_{\infty}(\Omega)\right)$ such that $Y \hookrightarrow X$, we say that the embedding $Y \hookrightarrow X$ is absolutely continuous (a.c. for short) if the elements of the closed unit ball of $Y, B_{Y}$, have the so-called Absolute Equicontinuity Norm Property in $X$, that is, if for every $\varepsilon>0$ there exists $\delta>0$ such that

$$
\left\|f \chi_{D}\right\|_{X}<\varepsilon, \quad \text { for all } f \in B_{Y}, \quad \text { whenever } \mu(D)<\delta .
$$

We want to mention that the interpolation of a more general notion like uniformly absolutely continuous operator has been investigated recently in [7].

\section{Necessary conditions for the absolute continuity}

We begin our study giving necessary conditions for the absolute continuity of an embedding between intermediate spaces with respect $\left(L_{1}(\Omega), L_{\infty}(\Omega)\right)$. Our first result establishes that whenever the embedding $Y \hookrightarrow X$ is a.c., the embedding between the corresponding associated spaces, $X^{\prime} \hookrightarrow Y^{\prime}$, also is a.c.

Lemma 3.1. Let $X$ and $Y$ be Banach spaces intermediate with respect to $\left(L_{1}(\Omega), L_{\infty}(\Omega)\right)$ such that $Y \hookrightarrow X$. If the embedding $Y \hookrightarrow X$ is a.c., then the associated embedding $X^{\prime} \hookrightarrow Y^{\prime}$ is a.c.

Proof. Assume that the embedding $Y \hookrightarrow X$ is a.c. Given $\varepsilon>0$, there exists $\delta>0$ such that

$$
\left\|f \chi_{D}\right\|_{X}<\varepsilon, \quad \text { for all } f \in B_{Y} \quad \text { and } \quad D \subset \Omega \quad \text { with } \mu(D)<\delta .
$$

Hence, for any $g \in B_{X^{\prime}}$, we have that

$$
\left\|g \chi_{D}\right\|_{Y^{\prime}}=\sup _{\|f\|_{Y} \leqslant 1} \int_{\Omega}\left|g \chi_{D} f\right| d \mu \leqslant \sup _{\|f\|_{Y} \leqslant 1}\|g\|_{X^{\prime}}\left\|f \chi_{D}\right\|_{X}<\varepsilon .
$$

and thus the embedding $X^{\prime} \hookrightarrow Y^{\prime}$ is a.c. 
Now we establish necessary conditions for an embedding to be a.c. in terms of the functions $\psi$ and $\rho$.

Theorem 3.2. Let $X$ and $Y$ be Banach spaces intermediate with respect to $\left(L_{1}(\Omega), L_{\infty}(\Omega)\right)$ such that $Y \hookrightarrow X$. If the embedding $Y \hookrightarrow X$ is a.c., then

$$
\lim _{t \rightarrow 0} \frac{\psi_{Y}(t)}{\psi_{X}(t)}=0
$$

Proof. By Lemma 3.1, the embedding $X^{\prime} \hookrightarrow Y^{\prime}$ is a.c. when $Y \hookrightarrow X$ is a.c. Therefore, given $\varepsilon>0$, there exists $\delta>0$ such that for any set $D \subset \Omega$ with $\mu(D) \leqslant t<\delta$ and any function $f \in B_{X^{\prime}}$ the inequality

$$
\left\|f \chi_{D}\right\|_{Y^{\prime}}<\varepsilon
$$

holds. In particular, for every set $D$ with $\mu(D) \leqslant t<\delta$, we derive that

$$
\left\|\frac{\chi_{D}}{\left\|\chi_{D}\right\|_{X^{\prime}}}\right\|_{Y^{\prime}}<\varepsilon
$$

Using (2.4) the theorem is proved.

The equivalence (2.5) allows to obtain in a straight way a necessary condition by means of the function $\rho$.

Theorem 3.3. Let $X$ and $Y$ be Banach spaces intermediate with respect to $\left(L_{1}(\Omega), L_{\infty}(\Omega)\right)$ such that $Y \hookrightarrow X$. If $Y \hookrightarrow X$ is an a.c. embedding, then

$$
\lim _{t \rightarrow 0} \frac{\rho_{Y}(t)}{\rho_{X}(t)}=0
$$

We also get the following.

Theorem 3.4. Let $X$ and $Y$ be Banach spaces intermediate with respect to $\left(L_{1}(\Omega), L_{\infty}(\Omega)\right)$ such that $Y \hookrightarrow X$. If the embedding $Y \hookrightarrow X$ is a.c., then

$$
\lim _{t \rightarrow 0} \frac{\rho_{Y}(t)}{\psi_{X}(t)}=0 .
$$

Proof. First note that for each $D \subset \Omega$ with $\mu(D)=t$, it follows that

$$
K\left(t, \chi_{D}\right)=K\left(t, \chi_{D} ; L_{1}(\Omega), L_{\infty}(\Omega)\right)=t
$$

and

$$
J\left(t, \chi_{D}\right)=J\left(t, \chi_{D} ; L_{1}(\Omega), L_{\infty}(\Omega)\right)=t .
$$

On the other hand, given $\varepsilon>0$, there exists $\delta>0$ such that $\left\|f \chi_{D}\right\|_{X}<\varepsilon$ for any function $f \in B_{Y}$ and any set $D$ with $\mu(D)=t<\delta$. Therefore,

$$
\frac{\rho_{Y}(t)}{\psi_{X}(t)}=\frac{\rho_{Y}(t)}{J\left(t, \chi_{D}\right)} \frac{K\left(t, \chi_{D}\right)}{\psi_{X}(t)} \leqslant\left\|\frac{\chi_{D}}{\left\|\chi_{D}\right\|_{Y}}\right\|_{X}<\varepsilon,
$$

and the theorem is proved. 
For the particular case when $X, Y$ are rearrangement invariant spaces the conditions (3.1), (3.2) and (3.3) turn out to be condition (3.1) in [11], given by means of the fundamental functions of the spaces $X$ and $Y$ :

$$
\lim _{t \rightarrow 0} \frac{\varphi_{X}(t)}{\varphi_{Y}(t)}=0
$$

The conditions (3.1), (3.2) and (3.3) are not equivalent, as it is shown in the following example.

Example 3.5. Consider the interval $[0, \infty)$ with the Lebesgue measure. We denote by $A$ the space of measurable functions on $[0, \infty)$ such that

$$
\|f\|_{A}=\sum_{n \geqslant 0}\|f\|_{L_{1}[2 n, 2 n+1)}+\sup _{n \geqslant 0}\|f\|_{L_{\infty}[2 n+1,2 n+2)} .
$$

It is clear that $L_{1}[0, \infty) \cap L_{\infty}[0, \infty) \hookrightarrow A \hookrightarrow L_{1}[0, \infty)+L_{\infty}[0, \infty)$. Indeed, if $f \in L_{1}[0, \infty) \cap L_{\infty}[0, \infty)$,

$$
\begin{aligned}
\|f\|_{A} & =\sum_{n \geqslant 0}\|f\|_{L_{1}[2 n, 2 n+1)}+\sup _{n \geqslant 0}\|f\|_{L_{\infty}[2 n+1,2 n+2)} \\
& \leqslant\|f\|_{L_{1}[0, \infty)}+\|f\|_{L_{\infty}[0, \infty)} \leqslant 2\|f\|_{L_{1}[0, \infty) \cap L_{\infty}[0, \infty)} .
\end{aligned}
$$

Analogously, if $f \in A$, let

$$
f_{0}(s)= \begin{cases}f(s), & \text { if } s \in[2 n, 2 n+1) \text { for } n \geqslant 0 \\ 0 & \text { in other case }\end{cases}
$$

and

$$
f_{1}(s)= \begin{cases}f(s), & \text { if } s \in[2 n+1,2 n+2) \text { for } n \geqslant 0 \\ 0 & \text { in other case. }\end{cases}
$$

Then,

$$
\begin{aligned}
\|f\|_{L_{1}[0, \infty)+L_{\infty}[0, \infty)} & \leqslant\left\|f_{0}\right\|_{L_{1}[0, \infty)}+\left\|f_{1}\right\|_{L_{\infty}[0, \infty)} \\
& =\sum_{n \geqslant 0}\|f\|_{L_{1}[2 n, 2 n+1)}+\sup _{n \geqslant 0}\|f\|_{L_{\infty}[2 n+1,2 n+2)}=\|f\|_{A} .
\end{aligned}
$$

Note that $A$ is not a rearrangement invariant space, because $\chi_{[0,1 / 2]}$ and $\chi_{[1,3 / 2]}$ are equimeasurable functions, but their norms are not equal.

On the other hand, if $D \subset[0, \infty)$ with $\operatorname{mes}(D) \leqslant t$, for each $n \geqslant 0$, we have

$$
\left\|\chi_{D}\right\|_{L_{1}[2 n, 2 n+1)} \leqslant \int_{0}^{\min \{1, t\}} d s
$$


and so

$$
\begin{aligned}
\left\|\chi_{D}\right\|_{A} & =\sum_{n \geqslant 0}\left\|\chi_{D}\right\|_{L_{1}[2 n, 2 n+1)}+\sup _{n \geqslant 0}\left\|\chi_{D}\right\|_{L_{\infty}[2 n+1,2 n+2)} \\
& \leqslant\left(\left[\frac{t}{2}\right]+1\right) \int_{0}^{\min \{1, t\}} d s+1 \leqslant \frac{t}{2}+2 \leqslant t+2,
\end{aligned}
$$

where $[x]$ stands for the integer part of the real number $x$. Therefore,

$$
\frac{t}{t+2} \leqslant \frac{t}{\sup \left\{\left\|\chi_{D}\right\|_{A} ; \operatorname{mes}(D) \leqslant t\right\}} .
$$

Moreover, for each $0<t<1$,

$$
\sup \left\{\left\|\chi_{D}\right\|_{A} ; \operatorname{mes}(D) \leqslant t\right\} \geqslant\left\|\chi_{G}\right\|_{A}, \quad \text { with } G=\left[0, \frac{t}{2}\right) \cup\left[2-\frac{t}{2}, 2\right) .
$$

In consequence,

$$
\sup \left\{\left\|\chi_{D}\right\|_{A} ; \operatorname{mes}(D) \leqslant t\right\} \geqslant\left\|\chi_{\left[0, \frac{t}{2}\right)}\right\|_{L_{1}[0,1)}+\left\|\chi_{\left[2-\frac{t}{2}, 2\right)}\right\|_{L_{\infty}[1,2)}=\frac{t}{2}+1
$$

and hence

$$
\frac{t}{\sup \left\{\left\|\chi_{D}\right\|_{A} ; \operatorname{mes}(D) \leqslant t\right\}} \leqslant 2 \frac{t}{t+2} .
$$

The equivalence (2.5) implies that

$$
\rho_{A}(t) \sim \frac{t}{t+2}, \quad 0<t<1
$$

Now let us get an estimate for the function $\psi_{A}(t)$. We know by $(2.4)$ that

$$
\psi_{A}(t)=\sup \left\{\left\|\chi_{D}\right\|_{A^{\prime}} ; \operatorname{mes}(D) \leqslant t\right\} .
$$

Let $D \subset[0, \infty)$ such that $\operatorname{mes}(D) \leqslant t$. Given $f \in A$, with $\|f\|_{A} \leqslant 1$, put

$$
f_{0}(s)= \begin{cases}f(s), & \text { if } s \in[2 n, 2 n+1) \text { for } n \geqslant 0 \\ 0 & \text { in other case }\end{cases}
$$

and

$$
f_{1}(s)= \begin{cases}f(s), & \text { if } s \in[2 n+1,2 n+2) \text { for } n \geqslant 0 \\ 0 & \text { in other case }\end{cases}
$$

Then

$$
\begin{aligned}
\int_{0}^{\infty}\left|f(s) \chi_{D}(s)\right| d s \leqslant & \int_{0}^{\infty}\left|f_{0}(s) \chi_{D}(s)\right| d s+\int_{0}^{\infty}\left|f_{1}(s) \chi_{D}(s)\right| d s \\
\leqslant & \sum_{n \geqslant 0}\left\|f_{0}\right\|_{L_{1}[2 n, 2 n+1)} \\
& +\sup _{n \geqslant 0}\left\|f_{1}\right\|_{L_{\infty}[2 n+1,2 n+2)} \int_{0}^{\infty} \chi_{D}(s) d s \\
\leqslant & \|f\|_{A}(1+t) \leqslant 1+t .
\end{aligned}
$$


Thus

$$
\left\|\chi_{D}\right\|_{A^{\prime}}=\sup \left\{\int_{0}^{\infty}\left|f(s) \chi_{D}(s)\right| d s ;\|f\|_{A} \leqslant 1\right\} \leqslant 1+t,
$$

and we have that $\psi_{A}(t) \leqslant 1+t$. In addition, given $0<t<1$, it holds that

$$
\psi_{A}(t)=\sup \left\{\left\|\chi_{D}\right\|_{A^{\prime}} ; \operatorname{mes}(D) \leqslant t\right\} \geqslant\left\|\chi_{G}\right\|_{A^{\prime}}, \quad \text { with } G=\left[0, \frac{t}{2}\right) \cup\left[2-\frac{t}{2}, 2\right) .
$$

But

$$
\left\|\chi_{G}\right\|_{A^{\prime}}=\sup \left\{\int_{0}^{\infty}\left|f(s) \chi_{G}(s)\right| d s ;\|f\|_{A} \leqslant 1\right\} \geqslant \int_{0}^{\infty} \frac{1}{t} \chi_{\left[0, \frac{t}{2}\right)}(s) \chi_{G}(s) d s=\frac{1}{2}
$$

and

$$
\left\|\chi_{G}\right\|_{A^{\prime}}=\sup \left\{\int_{0}^{\infty}\left|f(s) \chi_{G}(s)\right| d s ;\|f\|_{A} \leqslant 1\right\} \geqslant \int_{0}^{\infty} \chi_{[1,2)}(s) \chi_{G}(s) d s=\frac{t}{2} .
$$

Hence $1+t \leqslant 4 \psi_{A}(t)$ holds, and so

$$
\psi_{A}(t) \sim 1+t, \quad 0<t<1 .
$$

Using the former estimates for the functions $\psi_{A}$ and $\rho_{A}$, we can derive that neither $L_{1}[0, \infty) \cap L_{\infty}[0, \infty) \hookrightarrow A$ nor $A \hookrightarrow L_{1}[0, \infty)+L_{\infty}[0, \infty)$ are a.c. embeddings. In fact, it follows from $(2.2)$ that

$$
\lim _{t \rightarrow 0} \frac{\psi_{L_{1}[0, \infty) \cap L_{\infty}[0, \infty)}(t)}{\psi_{A}(t)}=\lim _{t \rightarrow 0} \frac{\rho_{L_{1}[0, \infty) \cap L_{\infty}[0, \infty)}(t)}{\psi_{A}(t)}=0,
$$

but

$$
\lim _{t \rightarrow 0} \frac{\rho_{L_{1}[0, \infty) \cap L_{\infty}[0, \infty)}(t)}{\rho_{A}(t)}>0
$$

Analogously, by (2.3),

$$
\lim _{t \rightarrow 0} \frac{\rho_{A}(t)}{\rho_{L_{1}[0, \infty)+L_{\infty}[0, \infty)}(t)}=\lim _{t \rightarrow 0} \frac{\rho_{A}(t)}{\psi_{L_{1}[0, \infty)+L_{\infty}[0, \infty)}(t)}=0,
$$

but

$$
\lim _{t \rightarrow 0} \frac{\psi_{A}(t)}{\psi_{L_{1}[0, \infty)+L_{\infty}[0, \infty)}(t)}>0
$$

A similar example for the case of the interval $[0,1]$ can be obtained using the intermediate space considered in [8], Example 2.4.

In addition, none of the conditions (3.1), (3.2) and (3.3) are sufficient to guarantee an a.c. embedding, not even when both spaces are rearrangement invariant spaces. 
Example 3.6. For $p>1$, let $M_{t^{1 / p}}$ be the Marcinkiewicz space on $[0,1]$ with fundamental function $\varphi_{M_{t^{1 / p}}}(t)=t^{1 / p}$ and $A_{p}$ be the space consisting of all measurable functions $f$ on $[0,1]$ for which the quasi-norm

$$
\|f\|_{A_{p}}=\sup _{0<t<1}\left(\log \frac{e}{t}\right)^{-1} \int_{t}^{1} s^{1 / p} f^{*}(s) \frac{d s}{s}
$$

is finite. It can be checked that $M_{t^{1 / p}} \hookrightarrow A_{p}$ is not an a.c. embedding (see [11], Example 3.4). However,

$$
\lim _{t \rightarrow 0} \frac{\psi_{M_{t^{1 / p}}}(t)}{\psi_{A_{p}}(t)}=\lim _{t \rightarrow 0} \frac{\rho_{M_{t^{1 / p}}}(t)}{\rho_{A_{p}}(t)}=\lim _{t \rightarrow 0} \frac{\rho_{M_{t^{1 / p}}}(t)}{\psi_{A_{p}}(t)}=\lim _{t \rightarrow 0} \frac{\varphi_{A_{p}}(t)}{\varphi_{M_{t^{1 / p}}}(t)}=0 .
$$

\section{Sufficient conditions for the absolute continuity}

In this section we provide sufficient conditions to assure the absolute continuity of an embedding between Banach spaces that are intermediate with respect to $\left(L_{1}(\Omega), L_{\infty}(\Omega)\right)$. The next result due to Pustylnik [15] (see also [14], Theorems 1.4.8 and 1.4.9.) will be useful for our aim. For the sake of completeness, we show the proof established in [14].

Theorem 4.1. Let $A$ be a Banach space intermediate with respect to the couple $\left(L_{1}(\Omega), L_{\infty}(\Omega)\right)$. Let $\theta(t)=t / \psi_{A}(t)$ and let $\gamma(t)$ be the least concave majorant of the function $t / \rho_{A}(t)$. Then

$$
\Lambda_{\gamma} \hookrightarrow A \hookrightarrow M_{\theta},
$$

with embedding constants no greater than one.

Proof. Since $K\left(t, f ; L_{1}(\Omega), L_{\infty}(\Omega)\right)=\int_{0}^{t} f^{*}(s) d s$, for any $f \in A$, it holds that

$$
\|f\|_{M_{\theta}}=\sup _{0<t<\infty}\left\{\frac{\theta(t)}{t} \int_{0}^{t} f^{*}(s) d s\right\}=\sup _{0<t<\infty}\left\{\frac{K(t, f)}{\psi_{A}(t)}\right\} \leqslant\|f\|_{A} .
$$

Hence, the embedding $A \hookrightarrow M_{\theta}$ holds.

On the other hand, for every $D \subset \Omega$ with $\mu(D)=t$, we have that

$$
\left\|\chi_{D}\right\|_{A} \leqslant \frac{J\left(t, \chi_{D}\right)}{\rho_{A}(t)}=\frac{\max \left\{\left\|\chi_{D}\right\|_{L_{1}(\Omega)}, t\left\|\chi_{D}\right\|_{L_{\infty}(\Omega)}\right\}}{\rho_{A}(t)}=\frac{t}{\rho_{A}(t)} \leqslant \gamma(t)=\left\|\chi_{D}\right\|_{\Lambda_{\gamma}} \text {. }
$$

The proof can be finished using analogous arguments to [12], Theorem II.5.5.

Next we establish sufficient conditions to guarantee an a.c. embedding.

Theorem 4.2. Let $X$ and $Y$ be Banach spaces intermediate with respect to $\left(L_{1}(\Omega), L_{\infty}(\Omega)\right)$. If $\lim _{t \rightarrow 0} t / \rho_{X}(t)=0$ and

$$
\int_{0}^{\mu(\Omega)} \frac{\psi_{Y}(t)}{t \rho_{X}(t)} d t<\infty
$$

then $Y \hookrightarrow X$ is a.c. 
Proof. Using Theorem 4.1, we consider the Marcinkiewicz space $M_{\theta}$ such that $Y \hookrightarrow M_{\theta}$, where $\theta(t)=t / \psi_{Y}(t)$. Analogously, let $\gamma(t)$ be the least concave majorant of the function $t / \rho_{X}(t)$ and $\Lambda_{\gamma}$ be the Lorentz space satisfying $\Lambda_{\gamma} \hookrightarrow$ $X$. By hypothesis, given any $\varepsilon>0$ there is $0<\delta<\mu(\Omega)$ such that (see [3], Lemma 1.3.4)

$$
\int_{0}^{\delta} \frac{\psi_{Y}(t)}{t \rho_{X}(t)} d t<\varepsilon .
$$

Now, using that $\gamma$ is a concave function (and thus $\gamma^{\prime}(t) \leqslant \gamma(t) / t$ ), we have that for any set $D$ with $\mu(D)<\delta$ and any function $f \in B_{M_{\theta}}$,

$$
\begin{aligned}
\left\|f \chi_{D}\right\|_{\Lambda_{\gamma}} & =\lim _{t \rightarrow 0} \gamma(t) \cdot\|f\|_{L_{\infty}(\Omega)}+\int_{0}^{\infty}\left(f \chi_{D}\right)^{*}(t) \gamma^{\prime}(t) d t \\
& =\int_{0}^{\infty}\left(f \chi_{D}\right)^{*}(t) \gamma^{\prime}(t) d t \leqslant \int_{0}^{\infty} f^{*}(t) \chi_{D}^{*}(t) \frac{\gamma(t)}{t} d t \\
& \lesssim \int_{0}^{\mu(D)} \frac{\psi_{Y}(t)}{t \rho_{X}(t)} d t<\varepsilon .
\end{aligned}
$$

This proves the embedding $M_{\theta} \hookrightarrow \Lambda_{\gamma}$ is a.c. Therefore, the embedding $Y \hookrightarrow X$ is also a.c.

Remark 4.3. We note that if $A$ is a rearrangement invariant space intermediate with respect to a Banach couple $\left(A_{0}, A_{1}\right)$, then $\lim _{t \rightarrow 0} t / \rho_{A}(t)=0$ holds whenever $A$ is not continuously embedded into $A_{1}^{\sim}$, the Gagliardo completion of $A_{1}$ in the sum $A_{0}+A_{1}$ (see [5], Lemma 3.8 (ii)). In particular, if $X$ is a rearrangement invariant space on $\Omega$, then $X$ is an intermediate space with respect to the couple $\left(L_{1}(\Omega), L_{\infty}(\Omega)\right)$, and so $\lim _{t \rightarrow 0} t / \rho_{X}(t)=0$ whenever $X$ is not continuously embedded into $L_{\infty}(\Omega)$.

As a direct consequence of Theorem 4.2 and the equalities (2.2) and (2.3) we obtain the following corollaries.

Corollary 4.4. Let $X$ be a Banach space of measurable functions on $[0,1]$ such that $L_{\infty}[0,1] \hookrightarrow X \hookrightarrow L_{1}[0,1]$.

a) If $\int_{0}^{1} \frac{1}{\rho_{X}(t)} d t<\infty$, then $L_{\infty}[0,1] \hookrightarrow X$ is an a.c. embedding.

b) If $\int_{0}^{1} \frac{\psi_{X}(t)}{t} d t<\infty$, then $X \hookrightarrow L_{1}[0,1]$ is an a.c. embedding.

Corollary 4.5. Let $X$ be a Banach space of measurable functions on $[0, \infty)$ such that $L_{1}[0, \infty) \cap L_{\infty}[0, \infty) \hookrightarrow X \hookrightarrow L_{1}[0, \infty)+L_{\infty}[0, \infty)$.

a) If $\int_{0}^{\infty} \frac{\min \{1, t\}}{t \rho_{X}(t)} d t<\infty$, then $L_{1}[0, \infty) \cap L_{\infty}[0, \infty) \hookrightarrow X$ is an a.c. embedding.

b) If $\int_{0}^{\infty} \frac{\psi_{X}(t)}{t \max \{1, t\}} d t<\infty$, then $X \hookrightarrow L_{1}[0, \infty)+L_{\infty}[0, \infty)$ is an a.c. embedding. 
With the aim of showing another example, we recall that the Cesáro function space $\operatorname{Ces}_{p}[0, \infty), 1<p<\infty$, consists of the classes of Lebesgue measurable functions on $[0, \infty)$ such that

$$
\|f\|_{\operatorname{Ces}_{p}[0, \infty)}=\left[\int_{0}^{\infty}\left(\frac{1}{x} \int_{0}^{x}|f(t)| d t\right)^{p} d x\right]^{1 / p}<\infty .
$$

The structure, geometry and other properties of these spaces have been studied by Astashkin and Maligranda in recent papers (see [2] and references therein). In particular, these authors have shown in [2], Theorem 1 , that $\operatorname{Ces}_{p}[0, \infty)$ is not a rearrangement invariant space.

Example 4.6. For $0<a<\infty$, we denote by $\operatorname{Ces}_{p}[0, \infty)_{\mid[0, a]}$ the subspace formed by those functions of $\operatorname{Ces}_{p}[0, \infty)$ with support in $[0, a]$. According to [2], Theorem 1 , the embeddings

$$
L_{\infty}[0, a] \hookrightarrow L_{p}[0, a] \hookrightarrow \operatorname{Ces}_{p}[0, \infty)_{\mid[0, a]} \hookrightarrow L_{1}[0, a]
$$

are continuous and so $\operatorname{Ces}_{p}[0, \infty)_{\mid[0, a]}$ is an intermediate space with respect to $\left(L_{1}[0, a], L_{\infty}[0, a]\right)$.

Let us give an estimate of the functions $\rho_{\operatorname{Ces}_{p}[0, \infty) \mid[0, a]}$ and $\psi_{\operatorname{Ces}_{p}[0, \infty)_{\mid[0, a]}}$. Assume that $0<t<a$. A straightforward computation yields that

$$
\sup \left\{\left\|\chi_{G}\right\|_{\operatorname{Ces}_{p}[0, \infty) \mid[0, a]} ; \operatorname{mes}(G) \leqslant t\right\}=\left(\frac{p}{p-1}\right)^{1 / p} t^{1 / p} .
$$

Then, by (2.5), we have that

$$
\rho_{\operatorname{Ces}_{p}[0, \infty)_{\mid[0, a]}}(t) \sim t^{1-1 / p} .
$$

In order to estimate $\psi_{\operatorname{Ces}_{p}[0, \infty) \mid[0, a]}(t)$ we shall use (2.4). Namely, for any subset $G$ with $\operatorname{mes}(G) \leqslant t$, it follows from [2], Theorem 2 , that

$$
\left.\left\|\chi_{G}\right\|_{\left(\operatorname{Ces}_{p}[0, \infty) \mid[0, a]\right.}\right)^{\prime}=\left\|\chi_{G} \chi_{[0, a]}\right\|_{\left(\operatorname{Ces}_{p}[0, \infty)\right)^{\prime}} \leqslant p^{\prime}\left\|\tilde{\chi}_{[0, a]}\right\|_{L_{p^{\prime}}[0, \infty)},
$$

where $p^{\prime}=\frac{p}{p-1}$ and

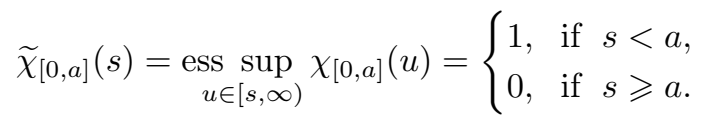

Thus, we get that $\psi_{\operatorname{Ces}_{p}[0, \infty)_{\mid[0, a]}}(t) \leqslant p^{\prime} a^{1 / p^{\prime}}$. Furthermore, applying again [2], Theorem 2, we have

$$
\begin{aligned}
\psi_{\operatorname{Ces}_{p}[0, \infty) \mid[0, a]}(t) & \left.\geqslant\left\|\chi_{[a-t, a]}\right\|_{\left(\operatorname{Ces}_{p}[0, \infty) \mid[0, a]\right.}\right)^{\prime} \\
& =\left\|\chi_{[a-t, a]}\right\|_{\left(\operatorname{Ces}_{p}[0, \infty)\right)^{\prime}} \geqslant \frac{1}{8}\left\|\widetilde{\chi}_{[a-t, a]}\right\|_{L_{p^{\prime}}[0, \infty)}=\frac{1}{8} a^{1 / p^{\prime}},
\end{aligned}
$$


since

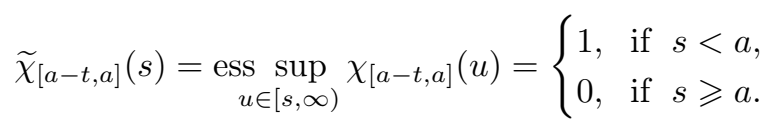

Therefore,

$$
\psi_{\mathrm{Ces}_{p}[0, \infty)_{\mid[0, a]}}(t) \sim a^{1-1 / p} .
$$

We derive from Theorem 4.2 that the embedding $L_{\infty}[0, a] \hookrightarrow \operatorname{Ces}_{p}[0, \infty)_{\mid[0, a]}$ is a.c. On the other hand, Theorem 3.2 yields that $\operatorname{Ces}_{p}[0, \infty)_{\mid[0, a]} \hookrightarrow L_{1}[0, a]$ is not an a.c. embedding. We also note that $L_{p}[0, a] \hookrightarrow \operatorname{Ces}_{p}[0, \infty)_{\mid[0, a]}$ is not absolutely continuous, by Theorem 3.3 .

\section{Absolute continuity: a quantitative estimate}

Let $X, Y$ be Banach spaces intermediate with respect to $\left(L_{1}(\Omega), L_{\infty}(\Omega)\right)$ such that $Y \hookrightarrow X$. We put, for each $0<h<\mu(\Omega)$,

$$
\sigma_{Y, X}(h)=\sup _{f \in B_{Y}} \sup _{D \subset \Omega, \mu(D) \leqslant h}\left\|f \chi_{D}\right\|_{X} .
$$

In order to guarantee that $\sigma_{Y, X}(h)$ is finite for every $h$, we shall assume that the spaces are lattices (in fact, it is enough to assume that $Y$ is a lattice).

The quantity $\sigma_{Y, X}(h)$ was introduced by J. Appell and E. De Pascale [1] in the context of $L_{p}$-spaces. The properties of the function $\sigma_{Y, X}$ in the setting of general rearrangement invariant spaces have been recently investigated by Evgeniy Pustylnik and the present authors in [11], showing in particular that $\sigma_{Y, X}$ yields a quantitative estimate of the degree of absolute continuity for the embedding $Y \hookrightarrow X$ between two rearrangement invariant spaces.

Next, we reproduce some important properties of this function that continue being valid for Banach lattices intermediate with respect to the couple $\left(L_{1}(\Omega), L_{\infty}(\Omega)\right)$ :

P1) The function $\sigma_{Y, X}$ is positive and increasing.

P2) Let $C_{Y, X}$ stand for the embedding constant of $Y \hookrightarrow X$. It holds that $\sup _{0<h<\mu(\Omega)} \sigma_{Y, X}(h)=C_{Y, X}$.

P3) The embedding $Y \hookrightarrow X$ is a.c. if and only if $\inf _{0<h<\mu(\Omega)} \sigma_{Y, X}(h)=0$.

P4) If $Z \hookrightarrow Y \hookrightarrow X$, then $\sigma_{Z, X}(h) \leqslant \sigma_{Z, Y}(h) \sigma_{Y, X}(h)$.

P5) It holds that $\sigma_{X^{\prime}, Y^{\prime}}(h) \leqslant \sigma_{Y, X}(h)$ and equality is fulfilled if $X$ and $Y$ have the Fatou property.

The proofs of these properties are easy. For instance, let $C_{Y, X}$ be the embedding constant of $Y \hookrightarrow X$. Then, for all $f \in B_{Y}$ and any set $D$ with $\mu(D)=h$, it holds that $\left\|f \chi_{D}\right\|_{X} \leqslant C_{Y, X}\left\|f \chi_{D}\right\|_{Y} \leqslant C_{Y, X}$ and so $\sup _{0<h<\mu(\Omega)} \sigma_{Y, X}(h) \leqslant C_{Y, X}$. The following argument yields the converse inequality. Given $\varepsilon>0$, there is $g \in B_{Y}$ such that $\|g\|_{X} \geqslant C_{Y, X}-\varepsilon$. Hence

$$
\sup _{0<h<\mu(\Omega)} \sigma_{Y, X}(h) \geqslant \sup _{0<h<\mu(\Omega)} \sup _{D \subset \Omega, \mu(D)=h}\left\|g \chi_{D}\right\|_{X}=\|g\|_{X} \geqslant C_{Y, X}-\varepsilon,
$$

and thus property P2) is proved. 
Next theorem provides an estimate of the function $\sigma_{Y, X}$.

Theorem 5.1. Let $X, Y$ be Banach lattices intermediate with respect to the couple $\left(L_{1}(\Omega), L_{\infty}(\Omega)\right)$. If $\lim _{t \rightarrow 0} t / \rho_{X}(t)=0$ and $\int_{0}^{\mu(\Omega)} \frac{\psi_{Y}(t)}{t \rho_{X}(t)} d t<\infty$, then

$$
\sigma_{Y, X}(h) \lesssim \int_{0}^{h} \frac{\psi_{Y}(t)}{t \rho_{X}(t)} d t, \quad 0<h<\mu(\Omega) .
$$

Proof. Reasoning as in the proof of Theorem 4.2, we can establish the inclusions

$$
Y \hookrightarrow M_{\theta} \hookrightarrow \Lambda_{\gamma} \hookrightarrow X
$$

where $\theta(t)=t / \psi_{Y}(t)$ and $\gamma(t)$ is the least concave majorant of the function $t / \rho_{X}(t)$. Moreover,

$$
\sigma_{M_{\theta}, \Lambda_{\gamma}}(h) \lesssim \int_{0}^{h} \frac{\psi_{Y}(t)}{t \rho_{X}(t)} d t, \quad \text { for any } 0<h<\mu(\Omega) .
$$

On the other hand, using property P2),

$$
\sigma_{Y, M_{\theta}}(h) \leqslant 1 \quad \text { and } \quad \sigma_{\Lambda_{\gamma}, X}(h) \leqslant 1 .
$$

Then, by property $\mathrm{P} 4)$,

$$
\sigma_{Y, X}(h) \leqslant \sigma_{M_{\theta}, \Lambda_{\gamma}}(h) \lesssim \int_{0}^{h} \frac{\psi_{Y}(t)}{t \rho_{X}(t)} d t, \quad 0<h<\mu(\Omega) .
$$

\section{Applications}

The function $\sigma_{Y, X}(h)$ was computed in [11] for the case of $L_{p}$-spaces on the interval $[0,1]$ with the Lebesgue measure. Precisely, for $p>q$, it holds that

$$
\sigma_{L_{p}, L_{q}}(h)=h^{\frac{1}{q}-\frac{1}{p}}, \quad h>0 .
$$

In particular, this estimate shows that the embedding

$$
L_{p}[0,1] \hookrightarrow L_{q}[0,1]
$$

is a.c. (see property $\mathrm{P} 3$ ) above).

We recall that if $E$ is a Banach space intermediate with respect to the couple $\left(L_{1}[0,1], L_{\infty}[0,1]\right)$, the inclusion indices of $E$ are defined by:

$$
\bar{\delta}_{E}=\sup \left\{p \geqslant 1 ; E \hookrightarrow L_{p}[0,1]\right\} \quad \text { and } \quad \bar{\gamma}_{E}=\inf \left\{p \leqslant \infty ; L_{p}[0,1] \hookrightarrow E\right\}
$$

Combining the absolute continuity of the embedding (6.1) together with the definition of inclusion indices, we obtain the following. 
Theorem 6.1. Let $X$ and $Y$ be Banach spaces of measurable functions on $[0,1]$ such that

$$
L_{\infty}[0,1] \hookrightarrow Y \hookrightarrow X \hookrightarrow L_{1}[0,1]
$$

If $\bar{\gamma}_{X}<\bar{\delta}_{Y}$, then $Y \hookrightarrow X$ is a.c.

As a consequence, we can estimate the degree of proximity between intermediate spaces for the couple $\left(L_{1}[0,1], L_{\infty}[0,1]\right)$ whose inclusion is not a.c.

Theorem 6.2. Let $X$ and $Y$ be Banach spaces of measurable functions on $[0,1]$ such that

$$
L_{\infty}[0,1] \hookrightarrow Y \hookrightarrow X \hookrightarrow L_{1}[0,1]
$$

Assume that $\bar{\delta}_{Y}=\bar{\gamma}_{Y}$ and $\bar{\delta}_{X}=\bar{\gamma}_{X}$. If the embedding $Y \hookrightarrow X$ is not a.c., then one of the following holds:

(i) $L_{\infty}[0,1] \subseteq Y \subseteq X \subseteq \bigcap_{1 \leqslant q<\infty} L_{q}[0,1]$,

(ii) $\bigcup_{1<q \leqslant \infty} L_{q}[0,1] \subseteq Y \subseteq X \subseteq L_{1}[0,1]$,

(iii) $\bigcup_{p<q \leqslant \infty} L_{q}[0,1] \subseteq Y \subseteq X \subseteq \bigcap_{1 \leqslant q<p} L_{q}[0,1]$, for some $1<p<\infty$.

Proof. If $\bar{\gamma}_{X}<\bar{\delta}_{Y}$, then the embedding $Y \hookrightarrow X$ would be a.c. Hence, it must be $\bar{\delta}_{Y} \leqslant \bar{\gamma}_{X}$. On the other hand, since $Y \hookrightarrow X$, it follows that $\bar{\gamma}_{X} \leqslant \bar{\gamma}_{Y}$. Then

$$
\bar{\delta}_{Y}=\bar{\gamma}_{Y}=\bar{\delta}_{X}=\bar{\gamma}_{X}
$$

Let $\bar{\delta}_{Y}=p$. If $p=\infty$, we obtain (i). When $p=1$, (ii) holds. If $1<p<\infty$, then we have (iii).

The indices $\bar{\delta}_{E}$ and $\bar{\gamma}_{E}$ above were defined, with the help of the scale of Lebesgue spaces $\left\{L_{p}[0,1]\right\}_{1 \leqslant p \leqslant \infty}$, for an intermediate space $E$ with respect to the couple $\left(L_{1}[0,1], L_{\infty}[0,1]\right)$. Recently, Cobos, Fernández-Cabrera, Hernández and Sánchez in [6] have extended this notion by considering indices defined by an abstract interpolation scale. Next we recall their precise definition. Let $\left(A_{0}, A_{1}\right)$ be an ordered couple, $A_{0} \hookrightarrow A_{1}$, and consider the complex interpolation scale for this couple, $\left\{A_{\theta}\right\}_{0 \leqslant \theta \leqslant 1}$, where $A_{\theta}=\left(A_{0}, A_{1}\right)_{[\theta]}$ (we refer to [4] and [12] for a comprehensive information about the complex interpolation method).

Definition 6.3. Given any intermediate space $A$ with respect to the ordered couple $\left(A_{0}, A_{1}\right)$, the indices of $A$ relative to the scale $\left\{A_{\theta}\right\}_{0 \leqslant \theta \leqslant 1}$ are defined by

$$
\delta_{A}=\sup \left\{\frac{1}{1-\theta}: A_{\theta} \hookrightarrow A\right\} \quad \text { and } \quad \gamma_{A}=\inf \left\{\frac{1}{1-\theta}: A \hookrightarrow A_{\theta}\right\} .
$$

Since $A_{0} \hookrightarrow A_{1}$, it follows from the properties of the complex interpolation method that $A_{\theta_{0}} \hookrightarrow A_{\theta_{1}}$ if and only if $\theta_{0}<\theta_{1}$, and so $1 \leqslant \delta_{A} \leqslant \gamma_{A} \leqslant \infty$. On the other hand, in the case of an intermediate space $A$ with respect to the couple $\left(L_{1}[0,1], L_{\infty}[0,1]\right)$ the indices $\delta_{A}, \gamma_{A}$ do not coincide with the indices $\bar{\delta}_{A}, \bar{\gamma}_{A}$ used 
before, but $1 / \delta_{A}+1 / \bar{\gamma}_{A}=1$ and $1 / \gamma_{A}+1 / \bar{\delta}_{A}=1$ (hence, $\delta_{A}=\gamma_{A}$ if and only if $\left.\bar{\delta}_{A}=\bar{\gamma}_{A}\right)$. These and other properties about the indices $\delta_{A}$ and $\gamma_{A}$ can be found in $[6]$.

We shall use the notion of indices defined by an abstract interpolation scale to estimate the grade of proximity between two intermediate spaces with respect to $\left(L_{1}(\Omega), L_{\infty}(\Omega)\right)$, in the case of the unbounded interval $\Omega=[0, \infty)$ with the Lebesgue measure.

It is well-known that if $A_{0}=L_{1}[0, \infty) \cap L_{\infty}[0, \infty)$ and $A_{1}=L_{1}[0, \infty)+$ $L_{\infty}[0, \infty)$, then

$$
A_{\theta}=\left(A_{0}, A_{1}\right)_{[\theta]}= \begin{cases}L_{p}[0, \infty) \cap L_{p^{\prime}}[0, \infty), \frac{1}{p}=1-\theta & \text { if } 0<\theta \leqslant 1 / 2, \\ L_{p}[0, \infty)+L_{p^{\prime}}[0, \infty), \frac{1}{p}=1-\theta & \text { if } 1 / 2 \leqslant \theta<1,\end{cases}
$$

where $1 / p+1 / p^{\prime}=1$.

Moreover, the embeddings between spaces of this interpolation scale are always a.c. embeddings. Actually, the spaces $\left\{A_{\theta}\right\}_{0<\theta<1}$ are rearrangement invariant spaces with the following fundamental functions:

$$
\begin{aligned}
& \varphi_{L_{p} \cap L_{p^{\prime}}}(t)=\max \left\{t^{1 / p}, t^{1 / p^{\prime}}\right\}, \quad t>0, \\
& \varphi_{L_{p}+L_{p^{\prime}}}(t)=\min \left\{t^{1 / p}, t^{1 / p^{\prime}}\right\}, \quad t>0 .
\end{aligned}
$$

Clearly, there are three different possibilities for the embedding $A_{\theta_{0}} \hookrightarrow A_{\theta_{1}}$ with respect to the position of the space $L_{2}[0, \infty)$ :

$$
\begin{aligned}
& A_{0} \hookrightarrow A_{\theta_{0}} \hookrightarrow A_{\theta_{1}} \hookrightarrow L_{2}[0, \infty) \hookrightarrow A_{1}, \\
& A_{0} \hookrightarrow A_{\theta_{0}} \hookrightarrow L_{2}[0, \infty) \hookrightarrow A_{\theta_{1}} \hookrightarrow A_{1}, \\
& A_{0} \hookrightarrow L_{2}[0, \infty) \hookrightarrow A_{\theta_{0}} \hookrightarrow A_{\theta_{1}} \hookrightarrow A_{1} .
\end{aligned}
$$

Thus, checking that the embedding $A_{\theta_{0}} \hookrightarrow A_{\theta_{1}}$ is a.c. amounts as much as checking that the corresponding embedding

$$
\begin{array}{ll}
L_{p}[0, \infty) \cap L_{p^{\prime}}[0, \infty) \hookrightarrow L_{q}[0, \infty) \cap L_{q^{\prime}}[0, \infty), & p<q \leqslant 2, \\
L_{p}[0, \infty) \cap L_{p^{\prime}}[0, \infty) \hookrightarrow L_{q}[0, \infty)+L_{q^{\prime}}[0, \infty), & p \leqslant 2<q, \\
L_{p}[0, \infty)+L_{p^{\prime}}[0, \infty) \hookrightarrow L_{q}[0, \infty)+L_{q^{\prime}}[0, \infty), & 2 \leqslant p<q,
\end{array}
$$

is a.c., which follows from Theorem 4.2. Therefore, the next result holds.

Theorem 6.4. Let $X$ and $Y$ be Banach spaces of measurable functions on $[0, \infty)$ such that

$$
L_{1}[0, \infty) \cap L_{\infty}[0, \infty) \hookrightarrow Y \hookrightarrow X \hookrightarrow L_{1}[0, \infty)+L_{\infty}[0, \infty)
$$

If $\gamma_{Y}<\delta_{X}$, then the embedding $Y \hookrightarrow X$ is a.c. 
The last result of this paper follows from a similar argument to the one of Theorem 6.2.

Theorem 6.5. Let $X$ and $Y$ be Banach spaces of measurable functions on $[0, \infty)$ such that

$$
L_{1}[0, \infty) \cap L_{\infty}[0, \infty) \hookrightarrow Y \hookrightarrow X \hookrightarrow L_{1}[0, \infty)+L_{\infty}[0, \infty)
$$

Assume $\delta_{Y}=\gamma_{Y}$ and $\delta_{X}=\gamma_{X}$. If the embedding $Y \hookrightarrow X$ is not a.c., then one of the following claims holds:

(i) $L_{1} \cap L_{\infty} \subseteq Y \subseteq X \subseteq\left(\bigcap_{1<p \leqslant 2} L_{p} \cap L_{p^{\prime}}\right) \cap\left(\bigcap_{2<p \leqslant \infty} L_{p}+L_{p^{\prime}}\right)$.

(ii) $\left(\bigcup_{1 \leqslant p \leqslant 2} L_{p} \cap L_{p^{\prime}}\right) \bigcup\left(\bigcup_{2<p<\infty} L_{p}+L_{p^{\prime}}\right) \subseteq Y \subseteq X \subseteq L_{1}+L_{\infty}$.

(iii) For some $1<q<\infty$,

$$
\begin{aligned}
& \left(\bigcup_{\substack{p<q \\
1 \leqslant p \leqslant 2}} L_{p} \cap L_{p^{\prime}}\right) \bigcup\left(\bigcup_{\substack{p<q \\
2<p}} L_{p}+L_{p^{\prime}}\right) \subseteq Y \subseteq X \\
& \qquad\left(\bigcap_{\substack{q<p \\
p \leqslant 2}} L_{p} \cap L_{p^{\prime}}\right) \cap\left(\bigcap_{\substack{q<p \\
2<p \leqslant \infty}} L_{p}+L_{p^{\prime}}\right) .
\end{aligned}
$$

Acknowledgements. The authors would like to thank Fernando Cobos for his helpful comments about this paper.

\section{References}

[1] J. Appell, E. De Pascale, Some parameters associated with the Hausdorff measure of noncompactness in spaces of measurable functions, Boll. Unione Mat. Ital. B 3 (1984), 497-515.

[2] S. Astashkin, L. Maligranda, Structure of Cesàro function spaces, Indag. Mathem., N.S. 20 (2009), 329-379.

[3] C. Bennett, R. Sharpley, Interpolation of Operators, Academic Press, 1988.

[4] J. Bergh, J. Löfström, Interpolation Spaces. An Introduction, Springer, 1976.

[5] F. Cobos, M. Cwikel, P. Matos, Best possible compactness results of LionsPeetre type, Proc. Edinburgh Math. Soc. 44 (2001), 153-172.

[6] F. Cobos, L.M. Fernández-Cabrera, F.L. Hernández, V.M. Sánchez, Indices defined by interpolation scales and applications, Proc. Royal Soc. Edinburgh 134 A (2004), 695-717.

[7] F. Cobos, A. Gogatishvili, B. Opic, L. Pick, Interpolation of uniformly absolutely continuous operators, Math. Nachr. 286 (2013), 579-599.

[8] F. Cobos, E. Pustylnik, On strictly singular and strictly cosingular embeddings between Banach lattices of functions, Math. Proc. Camb. Phil. Soc. 133 (2002), 183-190. 
[9] A.A. Dmitriev, The interpolation of one-dimensional operators, Voronež Gos. Univ. Trudy Naučn.-Issled. Inst. Mat. VGU Vyp. 11Sb. Statej Funkcional. Anal. i Prilozen 11 (1973), 31-43 (Russian).

[10] L.M. Fernández-Cabrera, Inclusion indices of function spaces and applications. Math. Proc. Camb. Phil. Soc. 136 (2004), 665-674.

[11] P. Fernández-Martínez, A. Manzano, E. Pustylnik, Absolutely continuous embeddings of rearrangement-invariant spaces, Mediterr. J. Math. 7 (2010), 539552.

[12] S.G. Krein, Ju. I. Petunin, E.M. Semenov, Interpolation of linear operators, Amer. Math. Soc., 1982.

[13] L. Maligranda, M. Mastylo, Notes on non-interpolation spaces, J. Approx. Theory 56 (1989), 333-347.

[14] P. Matos, Interpolação por meio de Espaços Intermédios Gerais de Operadores Compactos, Operadores Estrictamente Singulares e outros Ideais relacionados, $\mathrm{PhD}$, Universidade de Coimbra, 2000.

[15] E. Pustylnik, On maximal and minimal intermediate spaces, Ukrain. Mat. Zh. 29 (1977), 129-137 (English transl. in Ukrainian Math. J. 29 (1977), 102-107).

[16] E. Pustylnik, Embedding functions and thier role in interpolation theory, Abstract Appl. Anal. 1 (1996), 305-325.

[17] P.P. Zabreiko, Non-linear integral operators, Trudy Sem. Funkt. Anal. Voronezh 8 (1966), 3-148 (Russian).

Addresses: Pedro Fernández-Martínez: Departamento de Matemática Aplicada, Facultad de Informática, Universidad de Murcia, Campus de Espinardo, 30071 Espinardo (Murcia), Spain;

Antonio Manzano: Departamento de Matemáticas y Computación, Escuela Politécnica Superior, Universidad de Burgos, 09001 Burgos, Spain.

E-mail: pedrofdz@um.es, amanzano@ubu.es

Received: 9 October 2012; revised: 6 February 2013 